\title{
Body Constitution and Unhealthy Lifestyles in a Primary Care Population at High Cardiovascular Risk: New Insights for Health Management
}

\author{
Yi Wang ${ }^{1, *}$ \\ Xiao-Ya $W^{2, *}$ \\ Harry HX Wang (iD) ${ }^{1,3}$ \\ Yu-Ting $\mathrm{Li}^{4}$ \\ Yu Fu ${ }^{1,5}$ \\ Jia-Ji Wang 6,7 \\ Jose Hernandez 8,9 \\ Martin CS Wong $3,10,11$ \\ 'School of Public Health, Sun Yat-Sen University, \\ Guangzhou, People's Republic of China; ${ }^{2}$ Shipai \\ Community Health Centre, Tianhe District, \\ Guangzhou, People's Republic of China; ${ }^{3}$ C School \\ of Public Health and Primary Care, Faculty of \\ Medicine, The Chinese University of Hong Kong, \\ Sha Tin, Hong Kong Special Administrative Region; \\ ${ }^{4}$ State Key Laboratory of Ophthalmology, \\ Zhongshan Ophthalmic Center, Sun Yat-Sen \\ University, Guangzhou, People's Republic of China; \\ ${ }^{5}$ Melbourne School of Population and Global \\ Health, The University of Melbourne, Melbourne, \\ Australia; ${ }^{6}$ Guangdong-Provincial Primary \\ Healthcare Association, Guangdong, People's \\ Republic of China; ${ }^{7}$ School of Public Health, \\ Guangzhou Medical University, Guangzhou, \\ People's Republic of China; ${ }^{8}$ Faculty of Medicine \\ and Health, EDU, Digital Education Holdings Ltd. \\ Kalkara, Malta; 'Green Templeton College, \\ University of Oxford, Oxford, UK; ${ }^{10}$ School of \\ Public Health, Peking University, Beijing, People's \\ Republic of China; "'School of Public Health, The \\ Chinese Academy of Medical Sciences and Peking \\ Union Medical College, Beijing, People's Republic \\ of China
}

*These authors contributed equally to this work

Correspondence: Harry HX Wang School of Public Health, Sun Yat-Sen University, No. 74 Zhongshan Road 2, Guangzhou, 510080, People's Republic of China

Email wanghx27@mail.sysu.edu.cn; haoxiangwang@cuhk.edu.hk
Background: Adherence to lifestyle recommendations remains insufficient in cardiovascular (CV) health management globally. Body constitution, from the perspective of traditional Chinese medicine, is primarily influenced by an individual's internal metabolism and susceptibility to external pathogenic factors. Nevertheless, less is known about whether body constitutions may play a role in the presence of unhealthy lifestyles. We aimed to explore the associations between body constitutions and unhealthy lifestyles among Chinese individuals at high CV risk.

Methods: Computerised data were retrieved from a primary care population-based health record for all 1739 eligible individuals at high CV risk who attended routine check-up in an urbanised, medium-size district in Guangzhou, China. Unhealthy lifestyles were determined in accordance with guideline recommendations. The body constitution was assessed on the basis of physical signs, personality, body symptoms, and the susceptibility to environmental changes, following nationally standard procedure. Binary logistic regression analyses were performed using marginal standardisation method.

Results: The participants ranged in age from 20 to 96 years, with a mean age of 69.55 years. There were slightly more females than males (52.3\% vs $47.7 \%)$. Current smoking, regular drinking, and physical inactivity were most common. Participants with a body constitution of phlegm-and-dampness type (adjusted odds ratio $[\mathrm{aOR}]=1.999,95 \%$ confidence interval $[\mathrm{CI}]$ $=1.003-3.984 ; p=0.049$ ) tended to be current smokers, and those assessed with special diathesis type $(\mathrm{aOR}=2.166,95 \% \mathrm{CI}=1.029-4.559 ; p=0.042)$ had a higher likelihood of being regular drinkers. Having a body constitution type of blood stasis $(\mathrm{aOR}=1.375,95 \%$ $\mathrm{CI}=1.029-1.838 ; p=0.031)$ or qi deficient $(\mathrm{aOR}=1.711,95 \% \mathrm{CI}=1.080-2.709 ; p=0.022)$ was associated with physical inactivity.

Conclusion: Our findings add to current evidence suggesting that an individual's body constitution is closely related to the presence of unhealthy lifestyles. This offers new insights for health management through body constitution-based strategies to target those at high $\mathrm{CV}$ risk who need tailor-made attention in lifestyle modifications during routine primary care.

Keywords: body constitution, unhealthy lifestyles, association, primary care, cardiovascular health management

\section{Introduction}

Cardiovascular disease (CVD) represents enormous challenges to global population health. ${ }^{1}$ Evidence confirms that healthy lifestyle choices could substantially reduce cardiovascular $(\mathrm{CV})$ risks, ${ }^{2}$ and that effective lifestyle modifications can delay subsequent need for pharmacological treatment or augment the CV risk-lowering effect. ${ }^{3,4}$ 
Smoking cessation, reduction of alcohol consumption, and regular aerobic exercises may probably serve as the most effective and cornerstone approaches to tackle CVD. ${ }^{5}$

However, the constancy of lifestyle recommendations over time remains insufficient worldwide.$^{6-8}$ Barriers may include a lack of social support, insufficient knowledge, negative emotions or health beliefs, resource constraints, underestimation of risk perception, and unfavourable culture preferences. ${ }^{9,10}$ Beyond these commonly reported triggers, a wealth of literature has also documented the contribution of complementary and alternative medicine in the prevention and control of CVD. ${ }^{11,12}$ Body constitution, from the perspective of traditional Chinese medicine, is influenced by one's internal metabolism and susceptibility to external pathogenic factors, manifesting individual health state. It differentiates how individuals feel and behave, and ultimately affects how different bodies respond to causes of disease. ${ }^{13-15}$ Given the continuing challenges towards primary prevention and control of CVD, it remains largely unknown whether body constitutions may play a role in the presence of unhealthy lifestyles.

The study aimed to explore the associations between body constitutions and unhealthy lifestyles among primary care population at high CV risk. The study tested the null hypothesis that body constitutions were not associated with current smoking, regular drinking, or physical inactivity.

\section{Methods}

\section{Study Design and Data Source}

This study was a cross-sectional analysis of a Chinese population-based health record routinely managed in the context of the national basic public health (BPH) service delivery in primary care settings. ${ }^{16-18}$ The National Health Commission of China requires all community health centres (CHCs) to provide free-of-charge, annual check-up with lifestyle advices offered to adults diagnosed with hypertension or type 2 diabetes, and 65 -and-older population, who are primary care service users in the neighbourhood. The check-up reports were documented electronically by $\mathrm{CHC}$ public health staff. Computerised data were retrieved at a $\mathrm{CHC}$ in an urbanised, medium-size district in Guangzhou, China. The CHC serves a total of 22 neighbourhoods with a resident population size of 84 thousand, including approximately $5 \%$ who are eligible for annual check-up.

\section{Sample Size and Study Participants}

The minimum sample size of the source population, from which the target participants were drawn, was estimated based on a single proportion formula: $N=z^{2} p(1-p) / d^{2}$ where $z$ is the upper $\alpha / 2$ point of the normal distribution, $p$ is the population proportion, and $d$ is the precision. ${ }^{19}$ The parameters $(\alpha=0.05, z=1.96, p=0.103$, and $d=10 \%$ of $p$ ) were set based on a recent nationwide, population-based, large cohort study in China which reported a standardised prevalence of $10.3 \%$ of Chinese adults having high CV risk. ${ }^{20}$ From the above calculation, a source population having a sample size of 3346 would be sufficient to meet the criteria.

A total of 3852 individuals attended the annual checkup in 2019. The target participants were primary care population at high (including very high) $\mathrm{CV}$ risk assessed in accordance with the clinical guideline. ${ }^{21}$ Participants with any of the following were regarded as having high CV risk: (i) Systolic blood pressure (SBP)/diastolic blood pressure (DBP) 130-159/85-99 mmHg with co-presence of any physician-diagnosed hypertension-mediated organ damage, or $\geq 3 \mathrm{CV}$ risk factors, or chronic kidney disease grade $\geq 3$, or diabetes mellitus; (ii) SBP/DBP 160 $179 / 100-109 \mathrm{mmHg}$ with $\geq 1 \mathrm{CV}$ risk factors; or (iii) SBP/DBP $\geq 180 / 110 \mathrm{mmHg}$. Computerised data of a total of 1739 participants who fulfilled the eligibility criteria were retrieved from the routine BPH data platform.

\section{Study Variables and Data Collection}

A standard questionnaire derived from the national guideline was administered by the $\mathrm{CHC}$ healthcare staff to collect information on age, sex, and unhealthy lifestyles determined following national guidelines. ${ }^{16,21}$ Current smokers were defined as people who smoked at least one cigarette per day. ${ }^{21}$ Regular drinkers referred to those who frequently engaged in alcohol drinking for more than an equivalent of $25 \mathrm{~g}$ (for male) or $15 \mathrm{~g}$ (for female) of daily alcohol consumption; ${ }^{21}$ or for $\geq 4$ days per week. ${ }^{16}$ Participants who did not achieve $\geq 30$ minutes of active, moderate aerobic exercise in leisure time with faster heart beats and heavier breathing on 5 days per week on average were regarded as physical inactivity. ${ }^{21}$

BP was measured at a seated position using routinely validated automatic sphygmomanometers. The arm with the higher BP values was used. An average of two BP readings, 1-2 min apart, was recorded. The fasting plasma glucose was determined by enzymatic methods based on routine operating procedures. Lipid profiles were directly 
measured using an automated analyzer (Mindray BS-800 Analyzer, China). All the anthropometric measurements and laboratory tests were subject to internal quality control in accordance with clinical standard.

\section{Assessment of Body Constitution}

The assessment of body constitution was based on physical signs, personality, body symptoms, and the susceptibility to environmental changes. A standard set of questionnaire items with a 5-point Likert-type scale has been widely used in routine primary care following the guideline implementation. ${ }^{22}$ Individuals can be classified into one or more constitution types. All item scores reported by participants were inputted by the attending practitioner into a software for immediate results. A brief example of the body constitution assessment is shown in Appendix A (Supplementary Tables S1 and S2). The concise descriptions of the nine body constitution types are as follows:

- Neutral type: Individuals who are physically fit, emotionally stable, energetic, adaptable to environmental changes, and have good sleep habits and healthy appetite. This is the most balanced body constitution.

- Yin deficient type: Individuals who often complain about feeling warm in the palms of the hands and soles of the feet, especially at night, and have dry mouth and nose, dry stools or constipation, and an aversion to hot and dry weather.

- Yang deficient type: Individuals who have an aversion to cold and humidity, often complain about feeling cold at the hands, feet and abdomen, and feel unwell after eating cold food.

- Phlegm-dampness type: Individuals who are prone to excessive sebum secretions on the face and scalp, excessive phlegm production, heavy limbs, lethargy, chest tightness, cravings for food rich in fat and sugars, and have an aversion to humid and wet weather.

- Blood stasis type: Individuals who are prone to forgetfulness, irritability, bleeding disorders and abnormal growths in the body, and often have dull complexion and pigmentation on the skin, darkcoloured lips, unknown bruising, and an aversion to cold weather.

- Qi deficient type: Individuals who are prone to panting, easily fatigued, susceptible to the common cold or flu bug, and are easily affected by changes in weather or perspiring easily with little physical activity. These individuals usually take a longer time to recover from illness.

- Qi stagnation type: Individuals who are prone to mood swings, anxiety and depression, excessive sighing, palpitations, and have an aversion to autumn, winter and rainy seasons, and cope poorly to stress.

- Damp heat type: Individuals who are prone to excessive secretions or dampness at reproductive organs, feel fatigued and exhaustion; have acne on the face and neck, a sense of incomplete defecation or dry stools, and an aversion to warm and humid weather, and often complain of a bitter taste in the mouth.

- Special diathesis type (or intrinsic type): Individuals who have inherent sensitivity to certain drugs, foods, scents, or other allergens, and often have allergies that affect the respiratory system and skin, and are often sensitive to changes in temperature, weather, and seasons.

\section{Statistical Analyses}

Frequencies, percentages, and mean values with standard deviation (SD) were used for descriptive analysis. Binary logistic regression analysis was conducted to examine associations between body constitutions and each of the three commonly reported unhealthy lifestyles in the study. Following a conventional technique, the univariate analysis was performed in the first instance. Predictor variables with respect to demographics, lifestyles, anthropometric and clinical parameters that were statistically significant in the univariate model, as well as all body constitution types, were entered into the multivariable model. Sensitivity analyses for binary logistic regression models were also performed with all predictor variables included to examine whether the inclusion of additional variables could improve model fit. Both crude and adjusted odds ratios (aORs) were reported with 95\% confidence interval (CI). A $p$ value $<0.05$ was considered statistically significant. The predicted probabilities of unhealthy lifestyles were calculated using the marginal standardisation approach, which was considered appropriate for making inference on the overall source population where the study sample was drawn. ${ }^{23}$ All analyses were conducted in Stata 15.1 (StataCorp, TX).

\section{Ethics Consideration}

All participants provided written consent. Data anonymisation was performed by removing all subject identifiers from the dataset prior to data analysis. Ethics approval was 
granted from the School of Public Health Biomedical Research Ethics Review Committee at Sun Yat-Sen University (Ref: SYSUSPH-2019067) in accordance with the Declaration of Helsinki 2013.

\section{Results}

\section{Characteristics of Participants}

The analysis included data of 1739 primary care participants with complete information. Participants ranged in age from 20 to 96 years, with a mean age of 69.55 (SD 8.31) years. There were slightly more females than males (52.3\% vs $47.7 \%)$. Approximately one in ten $(9.3 \%)$ participants were current smokers, whilst nearly one fifth (17.4\%) were regular drinkers. More than one fourth (26.3\%) were physical inactive. The mean SBP and DBP were 143.09 (SD 12.97) $\mathrm{mmHg}$ and 84.45 (SD 9.63) $\mathrm{mmHg}$, respectively. The mean body mass index $\left(25.01 \mathrm{~kg} / \mathrm{m}^{2}\right)$ was slightly above the normal range ${ }^{21}$ $\left(18.5-23.9 \mathrm{~kg} / \mathrm{m}^{2}\right)$, and the control of plasma lipid levels with regard to the triglycerides $(1.75 \mathrm{mmol} / \mathrm{L}$; reference range $\left.^{21}<1.7 \mathrm{mmol} / \mathrm{L}\right)$ and plasma cholesterol levels $(5.43$ $\mathrm{mmol} / \mathrm{L}$; reference range ${ }^{21}<5.2 \mathrm{mmol} / \mathrm{L}$ ) were suboptimal (Table 1).

\section{Characteristics of Body Constitutions}

Among all participants, less than one fifth (17.0\%) had a neutral type of body constitution deemed as physically fit, emotionally stable, energetic, and adaptable to external environmental changes. The most prevalent type of nonneutral body constitution was phlegm-and-dampness, which was found in nearly two thirds $(61.7 \%)$ of participants, followed by the yin deficient type $(22.7 \%)$ and blood stasis type (17.4\%) (Figure 1).

\section{Factors Associated with Commonly Reported Unhealthy Lifestyles}

Fitting univariate and multivariable binary logistic regression models, current smoking was used as the dichotomous dependent variable and it was found that a phlegmand-dampness constitution type $(\mathrm{aOR}=1.999,95 \%$ $\mathrm{CI}=1.003-3.984 ; p=0.049)$ was associated with the presence of current smoking after adjusting for the effect of other predictor variables (Table 2). Similarly, in the regression model where regular drinking was used as the dichotomous dependent variable, it was found that participants with the special diathesis type $(\mathrm{aOR}=2.166,95 \%$ $\mathrm{CI}=1.029-4.559 ; p=0.042$ ) were more likely to be regular
Table I Demographics, Lifestyles and Clinical Parameters of Study Participants

\begin{tabular}{|l|r|}
\hline Variables & All Participants \\
\hline Demographics and lifestyles & \\
Age, mean (SD), years & $69.55(8.3 \mathrm{I})$ \\
Female, $\mathrm{n}(\%)$ & $910(52.3)$ \\
Current smoking, $\mathrm{n}(\%)$ & $\mathrm{I} 6 \mathrm{I}(9.3)$ \\
Regular drinking, $\mathrm{n}(\%)$ & $302(17.4)$ \\
Physical inactivity, $\mathrm{n}(\%)$ & $458(26.3)$ \\
\hline Anthropometric measurements & \\
Height, mean (SD), cm & $160.63(9.00)$ \\
Weight, mean (SD), kg & $64.74(10.88)$ \\
Waist, mean (SD), cm & $88.32(8.43)$ \\
BMl, mean (SD), kg/m ${ }^{2}$ & $25.01(3.08)$ \\
\hline Blood pressure & \\
Systolic BP, mean (SD), mmHg & $143.09(12.97)$ \\
Diastolic BP, mean (SD), mmHg & $84.45(9.63)$ \\
\hline Fasting lipid profile & \\
TC, mean (SD), mmol/L & \\
TG, mean (SD), mmol/L & $5.43(\mathrm{I} .12)$ \\
LDL cholesterol, mean (SD), mmol/L & $\mathrm{I} .75(\mathrm{I} .20)$ \\
HDL cholesterol, mean (SD), mmol/L & $3.29(0.91)$ \\
\hline Fasting blood glucose & $\mathrm{I} .37(0.37)$ \\
FPG, mean (SD), mmol/L & \\
\hline
\end{tabular}

Abbreviations: SD, standard deviation; BMI, body mass index; BP, blood pressure; TC, total cholesterol; TG, triglycerides; LDL, low density lipoprotein; HDL, high density lipoprotein; FPG, fasting plasma glucose.

drinkers (Table 3). It was also found that the blood stasis type $(\mathrm{aOR}=1.375,95 \% \mathrm{CI}=1.029-1.838 ; p=0.031)$ and $\mathrm{qi}$ deficient type $\quad(\mathrm{aOR}=1.711,95 \% \quad \mathrm{CI}=1.080-2.709$; $p=0.022$ ) were significantly associated with physical inactivity in the fitted univariate and multivariable binary logistic regression models (Table 4). Sensitivity analyses with all predictor variables included in the binary logistic regression models did not improve model fit. The strength and direction of the associations determined from the main analysis remained largely unchanged in the sensitivity analysis.

\section{Body Constitutions and Predicted Probabilities of Unhealthy Lifestyles}

The predicted probabilities (\%) of different types of body constitutions falling into categories of current smoking, regular drinking, or physical inactivity, respectively, were yielded from the aforementioned multivariable logistic regression models fitted in the main analysis (Tables 2-4). 


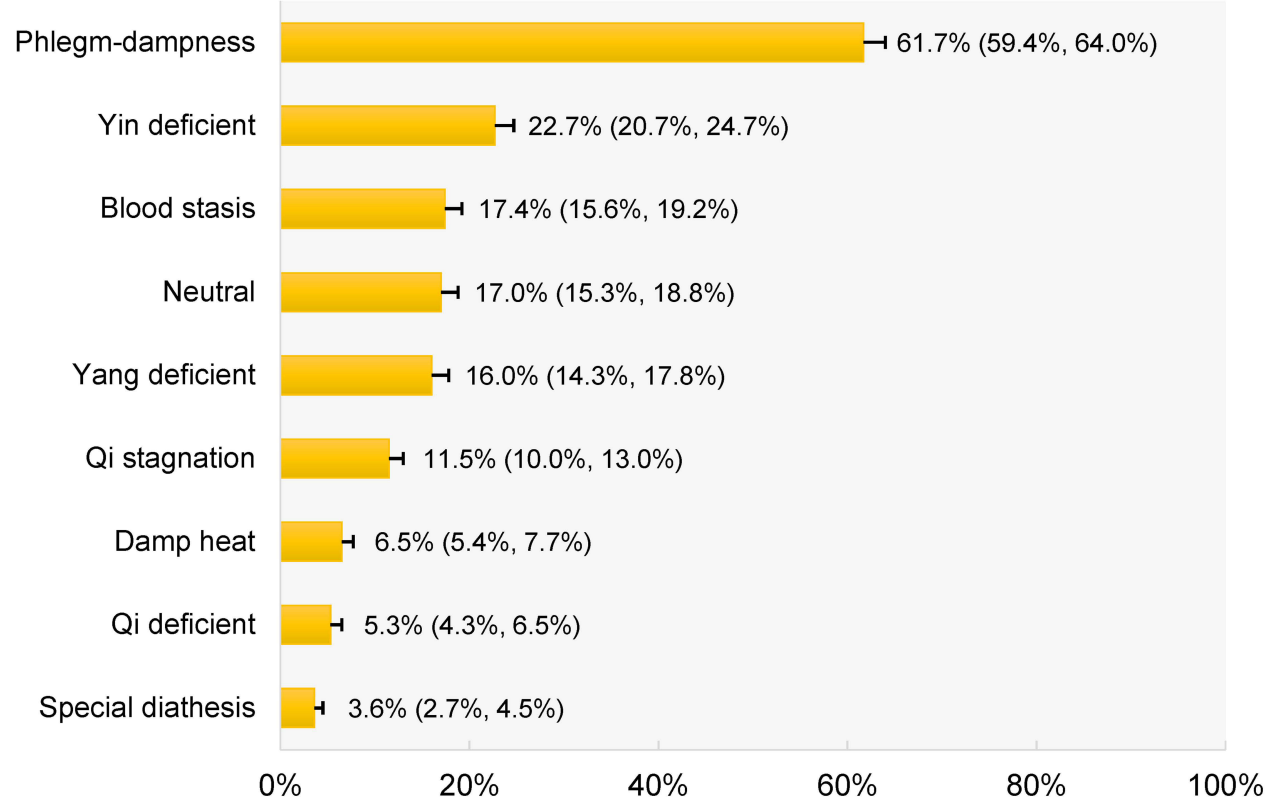

Figure I Profile of body constitutions of participants.

Notes: The sum of percentages exceeded $100 \%$ as one or more body constitutions may present concurrently within an individual. Error bars indicated the upper boundary of the $95 \%$ confidence intervals.

The raw data on predicted probabilities reported for three decimal places (Supplementary Table S3; Appendix B) were multiplied by 100 and rounded to the nearest integer. Results were presented in a three-colour scale drawing where all cells were coloured proportionally from green (ie, the lowest probability) to red (ie, the highest probability). For example, a qi deficient woman had a predicted probability of physical inactivity about 2 times that of a man with damp heat type of body constitution (Figure 2).

\section{Discussion}

\section{Main Findings}

This study adds to the evidence suggesting that unhealthy lifestyles inclusive of current smoking, regular drinking, and physical inactivity were closely related to individual's body constitutions. Participants with a body constitution of phlegm-and-dampness type tended to be current smokers, and those who were assessed with special diathesis type had a higher likelihood of being regular drinkers. The blood stasis type and qi deficient type of body constitutions were associated with inadequate physical activities.

\section{Relationship with Other Studies}

The relationships between unhealthy lifestyles and increased $\mathrm{CV}$ risks have been demonstrated in different populations worldwide. ${ }^{24-27}$ A number of CVD prevention programmes, such as the Public Health England CVD Ambitions, ${ }^{28}$ have placed equivalent emphasis on longterm lifestyle management and health behaviour change as core and fundamental components. ${ }^{29}$ Personalised support and access to stop smoking, physical activity and alcohol reduction services are actively available in the National Health Service Health Check to support early diagnosis and disease management. ${ }^{28}$ Compared to the US National Health Interview Survey (NHIS), ${ }^{30}$ the Dutch Monitoring Project on Risk Factors for Chronic Diseases (MORGEN), ${ }^{31}$ and the China Health and Retirement Longitudinal Study (CHARLS), ${ }^{32}$ our study population reported a relatively lower prevalence of smoking, drinking, and physical inactivity. This, however, implies that the use of body constitutions, when extended to the general population at large, may carry greater public health implications for informing unhealthy lifestyles.

Previous investigations have elaborated on the physiological and psychological mechanism by which body constitution relates to the development and prognosis of diseases. ${ }^{13-15,33}$ However, it is noteworthy that our main purpose is not to replicate existing findings or re-evaluate the relationship between body constitutions and CVD events per se. Instead, from the standpoint of preventive healthcare, our interest is to assess whether body constitutions may be associated with unhealthy lifestyles that are most prevalent in primary care population at high $\mathrm{CV}$ risk. 
Table 2 Binary Logistic Regression Analysis of Factors Associated with Current Smoking

\begin{tabular}{|c|c|c|c|c|}
\hline & \multicolumn{2}{|c|}{ Univariate Model } & \multicolumn{2}{|c|}{ Multivariable Model* } \\
\hline & cOR $(95 \% \mathrm{Cl})$ & $P$ & aOR $(95 \% \mathrm{CI})$ & $P$ \\
\hline Age, yrs* & $0.948(0.932,0.965)$ & $<0.001$ & $0.962(0.940,0.984)$ & 0.001 \\
\hline Female* & $0.005(0.001,0.033)$ & $<0.001$ & $0.005(0.001,0.039)$ & $<0.001$ \\
\hline Regular drinking* & $7.230(5.140,10.172)$ & $<0.001$ & $3.004(2.067,4.366)$ & $<0.001$ \\
\hline Physical inactivity* & $1.513(1.072,2.136)$ & 0.019 & $2.195(1.456,3.309)$ & $<0.001$ \\
\hline Waist, $\mathrm{cm}^{*}$ & $1.024(1.005,1.044)$ & 0.013 & $0.962(0.937,0.989)$ & 0.005 \\
\hline BMI, $\mathrm{kg} / \mathrm{m}^{2}$ & $0.978(0.927, \mathrm{I} .03 \mathrm{I})$ & 0.412 & - & - \\
\hline SBP, mmHg & $0.992(0.979,1.005)$ & 0.210 & - & - \\
\hline DBP, $\mathrm{mmH} \mathrm{g}^{*}$ & $1.033(1.016,1.050)$ & $<0.001$ & $0.985(0.962,1.007)$ & 0.180 \\
\hline $\mathrm{TG}, \mathrm{mmol} / \mathrm{L}$ & I.072 (0.952, I.207) & 0.251 & - & - \\
\hline LDL-C, $\mathrm{mmol} / \mathrm{L}$ & $0.930(0.776,1.116)$ & 0.437 & - & - \\
\hline $\mathrm{FPG}, \mathrm{mmol} / \mathrm{L}$ & $0.992(0.912,1.079)$ & 0.849 & - & - \\
\hline Neutral & $0.710(0.440,1.144)$ & 0.159 & $1.332(0.589,3.016)$ & 0.491 \\
\hline Yin deficient & I.186 (0.8I5, I.726) & 0.372 & $1.437(0.907,2.276)$ & 0.122 \\
\hline Yang deficient & $0.592(0.352,0.996)$ & 0.048 & $1.103(0.572,2.126)$ & 0.769 \\
\hline Phlegm-dampness & $1.972(1.350,2.880)$ & $<0.001$ & $1.999(1.003,3.984)$ & 0.049 \\
\hline Blood stasis & $0.862(0.552, \mathrm{I} .348)$ & 0.516 & $1.322(0.774,2.257)$ & 0.306 \\
\hline Qi deficient & $0.426(0.154,1.175)$ & 0.099 & $0.676(0.216,2.114)$ & 0.501 \\
\hline Qi stagnation & $0.429(0.215,0.855)$ & 0.016 & $0.597(0.272,1.31 \mathrm{I})$ & 0.198 \\
\hline Damp heat & $\mathrm{I} .06 \mathrm{I}(0.557,2.02 \mathrm{I})$ & 0.858 & I.I $44(0.528,2.478)$ & 0.733 \\
\hline Special diathesis & $0.667(0.239,1.863)$ & 0.440 & $1.079(0.329,3.540)$ & 0.900 \\
\hline
\end{tabular}

Notes: *Independent variables pertaining to demographics, lifestyles, anthropometric measures, and clinical parameters that were statistically significant in the univariate logistic regression model, as well as all body constitution types, were entered into the multivariable logistic regression model. Dependent variable: the presence of current smoking (yes versus no).

Abbreviations: cOR, crude odds ratio; aOR, adjusted odds ratio; $\mathrm{Cl}$, confidence interval; $\mathrm{BMI}$, body mass index; SBP, systolic blood pressure; DBP, diastolic blood pressure; TG, triglycerides; LDL-C, low density lipoprotein-cholesterol; FPG, fasting plasma glucose.

To our knowledge, such an association has not been thoroughly investigated. Our data exhibited a positive correlation between cigarette smoking and alcohol drinking. This is consistent with data from other populations worldwide, such as the Latin Americans and Caucasians, ${ }^{34-36}$ which may suggest a possible wider generalisability of our study findings.

\section{Meaning of the Study}

In our study, we speculate that the underlying nature of certain body constitutions might play a role in presenting character traits, which predispose one to unhealthy lifestyles. The qi deficient type of body constitution is often characterised by breathlessness, feeling tired easily, sweating spontaneously, perspiring easily with little physical activity, and a tendency to catch cold. This echoes the findings of our study, which indicated that qi deficient participants tended to have inadequate exercises. Recommendations therefore include efforts to ensure adequate sleep and keep warm at all times; avoid windy areas after strenuous exercises, and practice mild exercises such as brisk walks regularly. Meanwhile, our results also revealed that blood stasis individuals were prone to have physical inactivity. This might be explained by the difficulty in maintaining a smooth flow of energy in meridian pathways throughout the body. ${ }^{22}$ Advice offered to participants of this group may include carrying out activities, such as Tai Chi or dancing that help promote blood circulation.

The special diathesis constitution is often characterised by a predisposition of the body to allergens-induced conditions. ${ }^{13,14}$ The significant association observed between the special diathesis constitution and regular drinking is in line with a recent UK investigation, which reported that patients with inflammatory skin diseases tended to be frequent heavy alcohol drinkers, and were consequently at risk of developing alcohol use disorders. ${ }^{37}$ A bidirectional relationship may exist, meaning that regular drinking could be either a consequence or a trigger of psychological distress. Special diathesis participants may suffer from impaired quality of life due to the propensity to skin allergies, and this could promote alcohol abuse. Maintaining good personal hygiene and a wellventilated, clean living environment are equally important as countermeasures. 
Table 3 Binary Logistic Regression Analysis of Factors Associated with Regular Drinking

\begin{tabular}{|c|c|c|c|c|}
\hline & \multicolumn{2}{|c|}{ Univariate Model } & \multicolumn{2}{|c|}{ Multivariable Model* } \\
\hline & cOR $(95 \% \mathrm{Cl})$ & $P$ & aOR $(95 \% \mathrm{CI})$ & $P$ \\
\hline Age, yrs* & $0.95 \mathrm{I}(0.938,0.965)$ & $<0.001$ & $0.975(0.956,0.993)$ & 0.008 \\
\hline Female* & $0.099(0.070,0.141)$ & $<0.001$ & $0.162(0.109,0.241)$ & $<0.001$ \\
\hline Current smoking* & $7.334(5.485,9.805)$ & $<0.001$ & $3.023(2.185,4.182)$ & $<0.001$ \\
\hline Physical inactivity* & $0.729(0.54 \mathrm{I}, 0.98 \mathrm{I})$ & 0.037 & $0.720(0.510,1.015)$ & 0.061 \\
\hline Waist, $\mathrm{cm}^{*}$ & $1.035(1.020,1.051)$ & $<0.001$ & $1.004(0.984,1.025)$ & 0.691 \\
\hline BMI, $\mathrm{kg} / \mathrm{m}^{2}$ & $1.024(0.984,1.066)$ & 0.245 & - & - \\
\hline SBP, $\mathrm{mmHg} \mathrm{g}^{*}$ & $0.983(0.973,0.993)$ & 0.001 & $0.987(0.975,0.999)$ & 0.040 \\
\hline DBP, mmHg* & 1.035 (I.02I, I.048) & $<0.001$ & $1.015(0.996,1.035)$ & 0.132 \\
\hline $\mathrm{TG}, \mathrm{mmol} / \mathrm{L}$ & $1.078(0.982,1.184)$ & 0.115 & - & - \\
\hline LDL-C, $\mathrm{mmol} / \mathrm{L}$ & $0.927(0.806,1.065)$ & 0.284 & - & - \\
\hline $\mathrm{FPG}, \mathrm{mmol} / \mathrm{L}$ & $0.998(0.937,1.063)$ & 0.953 & - & - \\
\hline Neutral & $0.704(0.492,1.009)$ & 0.056 & $0.624(0.348,1.117)$ & 0.112 \\
\hline Yin deficient & $0.840(0.617,1.142)$ & 0.265 & $0.734(0.506,1.065)$ & 0.104 \\
\hline Yang deficient & $0.665(0.457,0.967)$ & 0.033 & $0.862(0.536,1.384)$ & 0.537 \\
\hline Phlegm-dampness & $1.609(1.223,2.117)$ & 0.001 & $0.844(0.522,1.366)$ & 0.490 \\
\hline Blood stasis & $0.804(0.570,1.136)$ & 0.216 & $\mathrm{I} .094(0.730, \mathrm{I} .64 \mathrm{I})$ & 0.664 \\
\hline Qi deficient & $0.625(0.329,1.188)$ & 0.152 & $0.887(0.424,1.859)$ & 0.751 \\
\hline Qi stagnation & $0.587(0.373,0.924)$ & 0.021 & $0.734(0.432,1.248)$ & 0.254 \\
\hline Damp heat & I.I $64(0.718,1.887)$ & 0.539 & $\mathrm{I} .287(0.72 \mathrm{I}, 2.297)$ & 0.393 \\
\hline Special diathesis & $1.276(0.683,2.383)$ & 0.445 & $2.166(1.029,4.559)$ & 0.042 \\
\hline
\end{tabular}

Notes: *Independent variables pertaining to demographics, lifestyles, anthropometric measures, and clinical parameters that were statistically significant in the univariate logistic regression model, as well as all body constitution types, were entered into the multivariable logistic regression model. Dependent variable: the presence of regular drinking (yes versus no).

Abbreviations: $\mathrm{COR}$, crude odds ratio; aOR, adjusted odds ratio; $\mathrm{Cl}$, confidence interval; $\mathrm{BMI}$, body mass index; SBP, systolic blood pressure; DBP, diastolic blood pressure; TG, triglycerides; LDL-C, low density lipoprotein-cholesterol; FPG, fasting plasma glucose.

Lethargy, heavy limbs, phlegm production, chest tightness, and an aversion to humid and wet weather are common characteristics of phlegm-and-dampness constitution. Our results revealed a close relationship between phlegm-and-dampness constitution and smoking. As smoking could lead to significant side effects such as phlegm production and chest tightness, symptoms seen in phlegm-and-dampness participants may be attributable to frequent tobacco consumption. It may be reasonable to speculate that participants who often feel lethargy or heavy limbs may experience enjoyment of smoking as a self-medication for boredom. ${ }^{38}$ They may necessitate close attention from primary care physicians to understand smoking initiation and relapse, and could be targeted as a priority group in smoking cessation interventions.

\section{Strengths and Weaknesses of the Study}

We collected data from a population sample of primary care service users of all ages to yield new insights for cardiovascular health management through body constitution-based strategies. Our results offer an innovative approach towards targeting those who need tailor-made attention in $\mathrm{CV}$ health-related lifestyle modifications in routine primary care. All data were retrieved from a computerised routine BPH platform to ensure data completeness and accuracy. However, our study had several limitations. First, the study was conducted in a single, medium-size district with a dense population. This may affect the generalisability of our findings, although we ensured a full age spectrum of participants. Second, we did not capture lifestyle behaviours that are not routinely evaluated in primary care, such as dietary intake, recreational drug use, and sleep history. Third, the reliance on self-report of lifestyles may be subject to recall bias, although we used dichotomous outcomes to minimise the extent to which information may deviate from objective measures. Fourth, factors such as education, socioeconomic status, and patients' engagement in healthcare may also play a role but were not captured in the study due to the absence of these variables in the data source. Moreover, a cause-and-effect relationship could not be established given the cross-sectional nature. The 
Table 4 Binary Logistic Regression Analysis of Factors Associated with Physical Inactivity

\begin{tabular}{|c|c|c|c|c|}
\hline & \multicolumn{2}{|c|}{ Univariate Model } & \multicolumn{2}{|c|}{ Multivariable Model* } \\
\hline & cOR $(95 \% \mathrm{Cl})$ & $P$ & aOR $(95 \% \mathrm{Cl})$ & $\mathbf{P}$ \\
\hline Age, yrs & $0.994(0.98 \mathrm{I}, \mathrm{I} .007)$ & 0.336 & - & - \\
\hline Female* & $1.387(1.118,1.721)$ & 0.003 & I.597 (I.220, 2.09I) & 0.001 \\
\hline Current smoking* & $1.513(1.072,2.136)$ & 0.019 & 2.279 (I.53।, 3.395) & $<0.001$ \\
\hline Regular drinking* & $0.729(0.54 I, 0.98 I)$ & 0.037 & $0.727(0.518,1.021)$ & 0.066 \\
\hline Waist, $\mathrm{cm}^{*}$ & $1.025(1.012,1.039)$ & $<0.001$ & $1.019(0.997,1.041)$ & 0.098 \\
\hline $\mathrm{BMI}, \mathrm{kg} / \mathrm{m}^{2 *}$ & $1.084(1.047,1.122)$ & $<0.001$ & $1.048(0.987,1.112)$ & 0.125 \\
\hline $\mathrm{SBP}, \mathrm{mmHg}$ & $1.004(0.995,1.012)$ & 0.386 & - & - \\
\hline $\mathrm{DBP}, \mathrm{mmHg}$ & $1.009(0.998,1.020)$ & 0.127 & - & - \\
\hline TG, mmol/L & $1.046(0.960,1.139)$ & 0.304 & - & - \\
\hline LDL-C, $\mathrm{mmol} / \mathrm{L}$ & $1.009(0.897,1.135)$ & 0.885 & - & - \\
\hline $\mathrm{FPG}, \mathrm{mmol} / \mathrm{L}$ & $1.010(0.957,1.065)$ & 0.722 & - & - \\
\hline Neutral & $0.791(0.589,1.063)$ & 0.120 & $1.507(0.975,2.330)$ & 0.065 \\
\hline Yin deficient & I.I 45 (0.89I, I.47I) & 0.290 & $1.234(0.924,1.649)$ & 0.155 \\
\hline Yang deficient & $1.309(0.989,1.731)$ & 0.059 & $1.390(0.993,1.944)$ & 0.055 \\
\hline Phlegm-dampness & $1.418(1.128,1.782)$ & 0.003 & $1.256(0.872,1.808)$ & 0.221 \\
\hline Blood stasis & $1.468(1.123,1.920)$ & 0.005 & $1.375(1.029,1.838)$ & 0.031 \\
\hline Qi deficient & $1.988(1.296,3.05 I)$ & 0.002 & I.7II (I.080, 2.709) & 0.022 \\
\hline Qi stagnation & I.277 $(0.926,1.760)$ & 0.136 & I.I $30(0.796,1.604)$ & 0.494 \\
\hline Damp heat & $1.096(0.717,1.675)$ & 0.671 & $0.843(0.535,1.326)$ & 0.459 \\
\hline Special diathesis & I.043 (0.59।, I.842) & 0.883 & $0.852(0.465,1.563)$ & 0.605 \\
\hline
\end{tabular}

Notes: *Independent variables pertaining to demographics, lifestyles, anthropometric measures, and clinical parameters that were statistically significant in the univariate logistic regression model, as well as all body constitution types, were entered into the multivariable logistic regression model. Dependent variable: the presence of physical inactivity (yes versus no).

Abbreviations: $\mathrm{COR}$, crude odds ratio; aOR, adjusted odds ratio; $\mathrm{Cl}$, confidence interval; $\mathrm{BMI}$, body mass index; SBP, systolic blood pressure; DBP, diastolic blood pressure; TG, triglycerides; LDL-C, low density lipoprotein-cholesterol; FPG, fasting plasma glucose.

possibility of the bidirectional relationships between body constitutions and unhealthy lifestyles cannot be ruled out, despite the fact that it carries social and behavioural implications to assume that unhealthy lifestyles serve as the consequences. This would necessitate future prospective, nationally representative studies that extend the coverage of participants with the assistance of remote symptom monitoring to capture various components of CV health-related lifestyles in long-term follow-up. Last but not least, our study results should be interpreted with caution as the concept of body constitution types may be debatable and current guidelines with respect to these concepts are based mainly on expert opinions, position statements and unpublished knowledge. For example, the term "blood stasis" is understood differently in the Western medicine compared to that in the traditional Chinese medicine, which may be due to physiological variations, terms used and language translations, and cultural differences in medical practices. ${ }^{39}$ However, it is worth noting that a comparison in the classification modalities of body constitution per se was not the aim of our study. Instead, we are more interested in determining whether body constitutions were associated with unhealthy lifestyles among individuals at high CV risk given the continuing challenges in primary prevention and control of CVD. ${ }^{2}$

\section{Implications for Clinical Practice}

Individuals' non-adherence to healthy lifestyles could jeopardise the efficiency of healthcare and predispose patients to $\mathrm{CV}$ disease progressing. The body constitutions determined from a rapid, easily performed assessment suggested a likelihood of individuals having unhealthy lifestyles. Furthermore, it provides early indications that could be linked to the mechanism for the difficulties experienced in maintaining recommended lifestyles. Alongside routine clinical assessment and regular health education, additional primary care efforts could be made to examine patients' body constitutions. This approach could be delivered in a manner whereby individuals at greater risks of unhealthy lifestyles are prioritised. Our work sheds light, furthermore, on the opportunity to ensure that individuals at high $\mathrm{CV}$ risk are 


\begin{tabular}{|c|c|c|c|c|c|c|}
\hline & MEN & & & & WOMEN & \\
\hline $\begin{array}{l}\text { Current } \\
\text { Smoking }\end{array}$ & $\begin{array}{l}\text { Regular } \\
\text { Drinking }\end{array}$ & $\begin{array}{l}\text { Physical } \\
\text { Inactivity }\end{array}$ & & $\begin{array}{c}\text { Current } \\
\text { Smoking }\end{array}$ & $\begin{array}{l}\text { Regular } \\
\text { Drinking }\end{array}$ & $\begin{array}{l}\text { Physical } \\
\text { Inactivity }\end{array}$ \\
\hline 23 & 25 & 30 & Neutral & $<1$ & 3 & 37 \\
\hline 23 & 27 & 26 & Yin deficient & $<1$ & 3 & 33 \\
\hline 22 & 29 & 29 & Yang deficient & $<1$ & 4 & 35 \\
\hline 24 & 31 & 24 & Phlegm-dampness & $<1$ & 4 & 32 \\
\hline 21 & 32 & 28 & Blood stasis & $<1$ & 5 & 35 \\
\hline 13 & 29 & 34 & Qi deficient & $<1$ & 4 & 41 \\
\hline 15 & 27 & 25 & Qi stagnation & $<1$ & 4 & 31 \\
\hline 22 & 38 & 21 & Damp heat & $<1$ & 6 & 27 \\
\hline \multirow[t]{2}{*}{22} & 46 & 21 & Special diathesis & $<1$ & 8 & 27 \\
\hline & & \multicolumn{5}{|c|}{$\begin{array}{l}\text { Interpretation } \\
\text { - } \quad \begin{array}{l}\text { A man wi } \\
\text { smoking } \\
\text { - }\end{array} \\
\text { A woman } \\
\text { drinking a } \\
\text { A woman } \\
\text { inactivity }\end{array}$} \\
\hline
\end{tabular}

Figure 2 Predicted probabilities of current smoking, regular drinking, and physical inactivity by body constitutions.

Notes: Predicted probabilities (\%) of unhealthy lifestyles were estimated using the marginal standardisation method from the multivariable logistic regression models, which adjusted for the effects of other predictor variables.

offered timely attention and tailor-made health management, helping to achieve the national and international goals in CVD prevention and control.

\section{Conclusion}

This study adds value to the evidence suggesting that an individual's body constitution is related to the presence of unhealthy lifestyles in a sample of primary care population with high CV risk. Participants with a body constitution of phlegm-and-dampness type tended to be current smokers, and those who were assessed with a special diathesis type had a higher likelihood of being regular drinkers. The blood stasis type and qi deficient type of body constitutions were associated with inadequate physical activities. Our results offer new insights for health management through body constitution-based strategies to target those who need tailor-made attention in lifestyle modifications during routine primary care.

\section{Data Sharing Statement}

The datasets used and analysed during the current study are available from the corresponding author on reasonable request.

\section{Acknowledgments}

We wish to acknowledge the tremendous support of the frontline primary care staff who were involved in the basic public health service delivery, body constitution assessment, healthcare record creation and routine check-up data documentation on the computerised data platform.

\section{Funding}

This work was supported by the National Natural Science Foundation of China (grant numbers 71673309, 71904212, and 72061137002); Science and Technology Development Fund of Guangdong Province (grant number 2016A020216006); Special Support Program of Guangdong Province (grant number 2017TQ04R749); Basic and Applied Basic Research Foundation of Guangdong Province (grant number 2019A1515011381); Traditional Chinese Medicine Research Program of Guangdong Province (grant number 20201055); and Higher Education Reform Project of Guangdong Province (grant number 20191206-20). The funders had no role in study design, data collection, data analysis, data interpretation, or writing of the report. 


\section{Disclosure}

The authors report no conflicts of interest in this work.

\section{References}

1. Roth GA, Mensah GA, Johnson CO, et al. Global burden of cardiovascular diseases and risk factors, 1990-2019: update from the GBD 2019 study. J Am Coll Cardiol. 2020;76(25):2982-3021.

2. Arnett DK, Khera A, Blumenthal RS. 2019 ACC/AHA guideline on the primary prevention of cardiovascular disease: part 1, lifestyle and behavioral factors. JAMA Cardiol. 2019;4(10):1043-1044. doi:10.1001/jamacardio.2019.2604

3. Marques-Vidal P. Comparison of lifestyle changes and pharmacological treatment on cardiovascular risk factors. Heart. 2020;106 (11):852-862. doi:10.1136/heartjnl-2019-316252

4. Williams B, Mancia G, Spiering W, et al. 2018 ESC/ESH guidelines for the management of arterial hypertension. Eur Heart J. 2018;39 (33):3021-3104.

5. Unger $\mathrm{T}$, Borghi $\mathrm{C}$, Charchar $\mathrm{F}$, et al. International Society of Hypertension global hypertension practice guidelines. J Hypertens. 2020;38(6):982-1004. doi:10.1097/HJH.0000000000002453

6. Kotseva K, De Backer G, De Bacquer D, et al. Primary prevention efforts are poorly developed in people at high cardiovascular risk: a report from the European Society of Cardiology EURObservational Research Programme EUROASPIRE V survey in 16 European countries. Eur J Prev Cardiol. 2021;28(4):370-379. doi:10.1177/ 2047487320908698

7. Gaziano TA. Lifestyle and cardiovascular disease: more work to do. $J \mathrm{Am}$ Coll Cardiol. 2017;69(9):1126-1128. doi:10.1016/j.jacc.2016.12.019

8. Cimminiello C. Never enough recommended. Beyond the AHA/ACC guideline on lifestyle and behavior for cardiovascular prevention. Eur J Intern Med. 2019;70:8-9. doi:10.1016/j.ejim.2019.10.021

9. Murray J, Fenton G, Honey S, Bara AC, Hill KM, House A. A qualitative synthesis of factors influencing maintenance of lifestyle behaviour change in individuals with high cardiovascular risk. $B M C$ Cardiovasc Disord. 2013;13:48. doi:10.1186/1471-2261-13-48

10. Alefan Q, Huwari D, Alshogran OY, Jarrah MI. Factors affecting hypertensive patients' compliance with healthy lifestyle. Patient Prefer Adherence. 2019;13:577-585. doi:10.2147/PPA.S198446

11. Rabito MJ, Kaye AD. Complementary and alternative medicine and cardiovascular disease: an evidence-based review. Evid Based Complement Alternat Med. 2013;2013:672097. doi:10.1155/2013/672097

12. Kohl WK, Dobos G, Cramer H. Conventional and complementary healthcare utilization among US adults with cardiovascular disease or cardiovascular risk factors: a nationally representative survey. $J \mathrm{Am}$ Heart Assoc. 2020;9(9):e014759. doi:10.1161/JAHA.119.014759

13. Li L, Yao H, Wang J, Li Y, Wang Q. The role of Chinese medicine in health maintenance and disease prevention: application of constitution theory. Am J Chin Med. 2019;47(3):495-506. doi:10.1142/ S0192415X19500253

14. Sun YZ, Zhao Y, Xue SA, Chen JP. The theory development of traditional Chinese medicine constitution: a review. $J$ Tradit Chin Med Sci. 2018;5(1):16-28.

15. Chan RY, Chien WT. Concepts of body constitution, health and sub-health from traditional Chinese medicine perspective. World $J$ Transl Med. 2013;2(3):56-66. doi:10.5528/wjtm.v2.i3.56

16. Department of Primary Health Care, National Health Commission, PRC; 2017, No.13 document. Standards for National Basic Public Health Services (third edition). Available from: http://www.nhc.gov. cn/jws/s3578/201703/d20c37e23e1f4c7db7b8e25f34473e1b.shtml. Accessed August 22, 2021.

17. Wang HHX, Wang JJ. Chapter 40 developing primary care in China. In: Griffiths SM, Tang JL, Yeoh EK, editors. Routledge Handbook of Global Public Health in Asia. London, UK: Routledge; 2014:584-600. doi: 10.4324/9781315818719.ch40
18. Wang HHX, Wang JJ, Wong SYS, Wong MCS, Mercer SW, Griffiths SM. The development of urban community health centres for strengthening primary care in China: a systematic literature review. Br Med Bull. 2015;116:139-153.

19. Thompson SK. Sampling. In: Wiley Series in Probability and Statistics. 3rd ed. ISBN 978-0-470-40231-3. New Jersey, USA: John Wiley \& Sons; 2012:60.

20. $\mathrm{Li} \mathrm{X}, \mathrm{Wu} \mathrm{C}, \mathrm{Lu} \mathrm{J}$, et al. Cardiovascular risk factors in China: a nationwide population-based cohort study. Lancet Public Health. 2020;5(12):e672-e681. doi:10.1016/S2468-2667(20) 30191-2

21. Liu LS; Writing Group of 2018 Chinese Guidelines for the Management of Hypertension. Chinese Hypertension League and Chinese Society of Cardiology. 2018 Chinese guidelines for the management of hypertension. Chin J Cardiovasc Med. 2019;24 (1):1-46.

22. China Association of Chinese Medicine. Classification and determination of body constitution in TCM: guideline no. ZYYXH/ T157-2009. Beijing, China: China Press of Traditional Chinese Medicine; 2009. ISBN 978-7-80231-614-0.

23. Muller CJ, MacLehose RF. Estimating predicted probabilities from logistic regression: different methods correspond to different target populations. Int J Epidemiol. 2014;43(3):962-970. doi:10.1093/ije/ dyu029

24. Piepoli MF, Hoes AW, Agewall S, et al. European Guidelines on cardiovascular disease prevention in clinical practice. Eur Heart $J$. 2016;37(29):2315-2381. doi:10.1093/eurheartj/ehw106

25. Zhang YB, Pan XF, Chen J, et al. Combined lifestyle factors, all-cause mortality and cardiovascular disease: a systematic review and meta-analysis of prospective cohort studies. $J$ Epidemiol Community Health. 2021;75(1):92-99.

26. Lv J, Yu C, Guo Y, et al. Adherence to healthy lifestyle and cardiovascular diseases in the Chinese population. J Am Coll Cardiol. 2017;69(9):1116-1125. doi:10.1016/j.jacc.2016.11.076

27. Barbaresko J, Rienks J, Nöthlings U. Lifestyle indices and cardiovascular disease risk: a meta-analysis. Am J Prev Med. 2018;55 (4):555-564. doi:10.1016/j.amepre.2018.04.046

28. Guidance - Health matters: preventing cardiovascular disease; 2019. Available from: https://www.gov.uk/government/publications/healthmatters-preventing-cardiovascular-disease/health-matters-preventingcardiovascular-disease\#cvd-ambitions-and-secondary-prevention. Accessed March 28, 2021.

29. Cowie A, Buckley J, Doherty P, et al. Standards and core components for cardiovascular disease prevention and rehabilitation. Heart. 2019;105(7):510-515. doi:10.1136/heartjnl-2018-314206

30. National Center for Health Statistics. Early release of selected estimates based on data from the 2018 National Health Interview Survey. Available from: https://www.cdc.gov/nchs/nhis/releases/ released201905.htm. Accessed March 28, 2021.

31. Hoevenaar-Blom MP, Spijkerman AM, Kromhout D, Verschuren WM. Sufficient sleep duration contributes to lower cardiovascular disease risk in addition to four traditional lifestyle factors: the MORGEN study. Eur J Prev Cardiol. 2014;21 (11):1367-1375. doi:10.1177/2047487313493057

32. Shao J, Wang X, Zou P, et al. Associating modifiable lifestyle factors with multimorbidity in community dwelling individuals from mainland China. Eur J Cardiovasc Nurs. 2021;20(6):556-564. doi:10.1093/eurjen/zvaa038

33. Liang X, Wang Q, Jiang Z, et al. Clinical research linking Traditional Chinese Medicine constitution types with diseases: a literature review of 1639 observational studies. J Tradit Chin Med. 2020;40 (4):690-702.

34. Britton M, Derrick JL, Shepherd JM, et al. Associations between alcohol consumption and smoking variables among Latinx daily smokers. Addict Behav. 2021;113:106672. doi:10.1016/j. addbeh.2020.106672 
35. Lynch KL, Twesten JE, Stern A, Augustson EM. Level of alcohol consumption and successful smoking cessation. Nicotine Tob Res. 2019;21(8):1058-1064. doi:10.1093/ntr/nty 142

36. Holahan CJ, Brennan PL, Schutte KK, Holahan CK, Hixon JG, Moos RH. Drinking level versus drinking pattern and cigarette smoking among older adults. Alcohol Clin Exp Res. 2018;42(4):795-802. doi:10.1111/acer.13607

37. Al-Jefri K, Newbury-Birch D, Muirhead CR, et al. High prevalence of alcohol use disorders in patients with inflammatory skin diseases. Br J Dermatol. 2017;177(3):837-844. doi:10.1111/bjd.15497
38. Twyman L, Bonevski B, Paul C, Bryant J. Perceived barriers to smoking cessation in selected vulnerable groups: a systematic review of the qualitative and quantitative literature. BMJ Open. 2014;4(12): e006414. doi:10.1136/bmjopen-2014-006414

39. Birch S, Alraek T, Lee MS, Lee JA, Kim TH. Understanding blood stasis in traditional East Asian medicine: a comparison of Asian and Western sources. Eur J Integr Med. 2021;44:101341. doi:10.1016/j. eujim.2021.101341

\section{Publish your work in this journal}

The International Journal of General Medicine is an international, peer-reviewed open-access journal that focuses on general and internal medicine, pathogenesis, epidemiology, diagnosis, monitoring and treatment protocols. The journal is characterized by the rapid reporting of reviews, original research and clinical studies across all disease areas. The manuscript management system is completely online and includes a very quick and fair peer-review system, which is all easy to use. Visit http://www.dovepress.com/ testimonials.php to read real quotes from published authors.

Submit your manuscript here: https://www.dovepress.com/international-journal-of-general-medicine-journal 